\title{
Peso bajo, sobrepeso, obesidad y crecimiento en adolescentes en el Perú 2009-2010
}

\author{
Low weight, overweight, obesity and growth in adolescents in Peru 2009-2010
}

\author{
Jaime Pajuelo R , José Sánchez-Abanto², Doris Álvarez-Dongo², \\ Carolina Tarqui-Mamani², Rosa Agüero Zamora ${ }^{3}$ \\ Instituto de Investigaciones Clínicas, Facultad de Medicina, Universidad Nacional Mayor de San Marcos, Lima, Perú \\ ${ }^{2}$ Centro Nacional de Alimentación (CENAN), Instituto Nacional de Salud, Perú \\ ${ }^{3}$ Servicio de Endocrinología. Hospital Nacional Dos de Mayo, Lima, Perú
}

\begin{abstract}
Resumen
Introducción: El grupo de adolescentes representan, de alguna manera, la quinta parte de la población y no se encuentran al margen de los problemas nutricionales. Objetivos: Conocer la situación nutricional, el crecimiento y algunos factores determinantes, en adolescentes en el Perú. Diseño: Estudio descriptivo, observacional, transversal. Participantes: Adolescentes. Intervenciones: En 14753 adolescentes de 10 a 19 años se obtuvo el peso, talla, índice de masa corporal (IMC). Se utilizó la referencia percentilar de Must y los puntajes Z de OMS. Principales medidas de resultados: Promedios y DE. Prevalencias IC 95\%. OR IC 95\%. Chi-cuadrado y regresión múltiple. Resultados: Se encontró prevalencias de 2,6; 5,9; 79; 9,3 y 3,2 (Must); 0,2; 1,1; 82,6; 12,5; 3,5\% (OMS) de déficit, peso bajo, normal, sobrepeso y obesidad, respectivamente. Predominó el sobrepeso-obesidad en áreas urbanas, en los no pobres, en la costa, selva y Lima Metropolitana, en los que vivían por debajo de $3000 \mathrm{msnm}$. Los factores de riesgo para el déficit-bajo peso fueron: género masculino, pobres extremos, pobres no extremos, vivir en la costa norte y sierra centro, y como factor protector los que estaban por debajo de los $1000 \mathrm{msnm}$. Los factores de riesgo del sobrepeso-obesidad fueron el vivir en áreas urbanas, costa sur y por debajo de los $1000 \mathrm{msnm}$, los que vivían entre los 1000 y $2999 \mathrm{msnm}$; y los factores de protección fueron el ser pobre extremo, el pobre no extremo, vivir en costa norte, sierra norte, sierra centro y sierra sur y selva. El $28,5 \%$ presentó retardo de crecimiento (adolescentes de 10 a 17 años). Los factores de riesgo del retardo de crecimiento fueron: vivir en áreas rurales, en la mayoría de los dominios geográficos excepto la costa sur, los niveles de pobreza (extrema y no extrema), los niveles de altitud (1 000 a 2999 y más de $3000 \mathrm{msnm}$ ). Conclusiones: El sobrepeso-obesidad es el problema de mayor magnitud en los adolescentes estudiados y confirma su tendencia al incremento en función del tiempo. La talla alcanzada dista de ser satisfactoria y refleja que aún mantenemos necesidades básicas insatisfechas.
\end{abstract}

Palabras clave: Adolescentes, nutrición, crecimiento, factores.

\section{Abstract}

Introduction: Teenagers represent somehow a fifth of the population and are not away from nutritional problems. Objectives: To determine the nutritional status, growth and some determining factors in adolescents in Peru. Design: Descriptive, observational, transversal study. Participants: Adolescents. Interventions: Weight, height, body mass index were obtained from 14753 adolescents 10-19 yearold. Must percentile reference and WHO Z score were used. Main outcome measures: Averages and DE, prevalence $\mathrm{Cl} 95 \%, \mathrm{OR}$ IC 95\%, chi-square and multiple regressions were obtained. Results: Prevalence of deficit, underweight, normal, overweight and obesity found respectively were $2.6 ; 5.9 ; 79 ; 9.3$ and 3.2 (Must); $0.2 ; 1.1 ; 82.6 ; 12.5 ; 3.5 \%$ (WHO). Overweight-obesity predominated in urban areas, in non-poor areas, the coast, jungle and Metropolitan Lima,and in those lived below 3000 masl. Risk factors for deficitunderweight were: male gender, extremely poor, non-extremely poor, living in the northern coast and central sierra; and as protective factor living below 1000 masl. Risk factors for overweight-obesity were living in urban areas, southern coast and below 1000 masl, and living between 1000 and 2999 meters; and protective factors were being extremely poor, and not extremely poor, living in the northern coast, northern sierra, central sierra, southern sierra and in the jungle. Growth retardation was found in 28.5\% of adolescents 10 to 17 years. Risk factors for growth retardation were living in rural areas, in most geographic domains except the southern coast, extreme and non-extreme poverty levels, altitude $1000-2999$ and above 3000 masl. Conclusions: Overweight-obesity was a problem of greater magnitude and confirmed its tendency to increase with time. Height attained was far from satisfactory and reflected maintenance of unsatisfied basic needs.

Keywords: Adolescents, nutrition, growth, factors.

An Fac med. 2015;76(2):147-54 / dx.doi.org/10.15381/anales.v76i2.11141 


\section{INTRODUCCIÓN}

La Organización Mundial de la Salud (OMS) define al grupo adolescente como aquel que está comprendido entre $\operatorname{los} 10$ a 19 años ${ }^{(1)}$. La adolescencia es el tránsito de la niñez a la vida adulta, siendo un periodo de enormes cambios en lo fisiológico, cognitivo y psicosocial, que de alguna manera son influenciados por cambios hormonales y socioambientales. El 20\% de la talla adulta y $25 \%$ del peso son alcanzados durante la adolescencia ${ }^{(2)}$.

En nuestro país y para el año 2010, los adolescentes alcanzaron casi $6 \mathrm{mi}$ llones, lo que representa de alguna manera la quinta parte de la población total ${ }^{(3)}$. Históricamente este grupo no ha sido considerado vulnerable, lo que significa que las políticas de salud no los ha tomado en cuenta como grupo objetivo, razón por lo cual existen pocos estudios para conocer su problemática.

Sin embargo, todos los cambios que se están dando en el mundo hace que los problemas nutricionales, como consecuencia de los cambios en los patrones alimentarios y la cada vez mayor inactividad física, merezcan una mayor atención a este grupo poblacional. El conocer su estado nutricional y el aporte que da en el crecimiento alcanzado, son los dos pilares que deben ser atendidos.

El primer estudio nutricional a nivel nacional fue realizado el año $1975^{(4)}$. En ese año, la población adolescente (10 a 19 años) llegaba a 3,5 millones. El estudio encontró $6,6 \%$ de sobrepeso y $1,3 \%$ de obesidad, mientras que el déficit alcanzaba $5,1 \%$ y el peso bajo $8,2 \%$, siendo el grupo femenino el más afectado. En esta época era más prevalente el déficit y peso bajo en relación a su contraparte.

En la década de 2000, un estudio llevado a cabo por el Ministerio de Educación, en población de 10 a 14 años, encontró en el género femenino 8,6\% de peso bajo/déficit y $18,6 \%$ de sobrepeso/obesidad, y en el masculino 9,2 y $9,7 \%$, respectivamente; el promedio de talla fue $1,31 \mathrm{~m}$ a los 10 años y $1,41 \mathrm{~m}$ a los 14 años ${ }^{(5)}$.

Posteriormente, un estudio dio una prevalencia de déficit y peso bajo de 0 y 1,3\% en Lima, 2,5 y 4,3\% en Trujillo, 6,2 y $10,3 \%$ en Huancavelica y 0,2 y $2,2 \%$ en Jauja, respectivamente. En lo que se refiere al sobrepeso y la obesidad, las prevalencias fueron 20,8 y $18,8 \%$ en Lima, 14,3 y $5,6 \%$ en Trujillo, 2,9 y $0,4 \%$ en Huancavelica y 5,6 y $2,0 \%$ en Jauja. Si bien este no fue un estudio nacional, se puede percibir que, con excepción de Huancavelica, se ha dado un cambio y ahora prima el sobrepeso y obesidad. En lo que se refiere al crecimiento alcanzado, el promedio de talla del grupo de 10 a 15 años fue $1,57 \mathrm{~m}$ en Lima y 1,38 m en Huancavelica ${ }^{(6)}$.

La obesidad en la infancia y adolescencia es un factor pronóstico de obesidad en el adulto. Las personas que tenían un índice de masa corporal (IMC) por encima del 60 percentil tienen prevalencia de sobrepeso a los 35 años, en hombres $34 \%$ y en mujeres $37 \%{ }^{(7)}$.

El objetivo del presente trabajo es conocer la situación del estado nutricional y el crecimiento alcanzado en la población adolescente e identificar algunos factores determinantes.

\section{MÉTODOS}

El presente estudio se ha hecho en base a la información de la Encuesta Nacional de Hogares (ENAHO) 2009-2010, específicamente a lo que se conoce como Componente Nutricional, llevado a cabo conjuntamente con el Centro Nacional de Alimentación y Nutrición (CENAN). La metodología del diseño y del muestreo se puede revisar ampliamente en el trabajo original ${ }^{(8)}$.

Se estudió 14753 adolescentes de 10 a 19 años; el 50,1\% correspondió al género masculino. A todos se les tomó el peso y la talla; estas mediciones antropométricas se realizaron de acuerdo a recomendaciones dadas por el CE$\mathrm{NAN}^{(9)}$.
El diagnóstico nutricional se hizo mediante el índice de masa corporal (IMC), la clasificación percentilar y con la población referencial de Must y col (NCSH) ${ }^{(10)}$, tomada del National Health and Nutrition Examination Survey (NHANES I). Los criterios diagnósticos fueron por debajo de 5 entre 5 y por debajo de 15 , de 15 a por debajo de 85 , de 85 a por debajo de 95 y del 95 percentil a más, para identificar déficit, peso bajo, normal, sobrepeso y obesidad, respectivamente. También se ha usado la referencia de la Organización Mundial de la Salud (OMS), con los puntajes $Z^{(11)}$.

El retardo de crecimiento fue diagnosticado utilizando la referencia del $\mathrm{NCSH}^{(12)}$, con el criterio diagnóstico de menor de menos 2 desvío estándar. Solo se utilizó las edades entre 10 y 17 años, por razones de la referencia. Las variables estudiadas para ver asociación fueron: género, área de residencia, dominios, nivel de altitud y nivel de pobreza.

La pobreza se midió por un enfoque monetario. Se consideró hogar pobre cuando el gasto per cápita era inferior a la línea de pobreza (hogares que cubren sus necesidades en alimentación, pero no cubren sus necesidades en otros bienes y servicios como educación, salud, vestido, calzado, entre otros), y hogar pobre extremo, cuando el gasto per cápita era inferior a una línea de pobreza extrema (hogares que no pueden cubrir sus gastos en alimentos ni sus gastos en otros bienes y servicios) ${ }^{(13)}$.

Los datos fueron analizados con el programa estadístico SPSS 18.0 y son presentados como promedios con sus respectivos desvíos estándar; asimismo, prevalencias e IC al 95\%, con un nivel de significancia de $p<0,05$. Para determinar la significación, se usó la prueba chi cuadrado para las variables categóricas.

La asociación de las variables fue determinada por medio del análisis para muestras complejas, utilizando la regresión multivariada, con los mismos IC y nivel de significancia que lo expuesto anteriormente. 
Tabla 1. Distribución del estado nutricional según estándar OMS y MUST (NCSH) y sexo en adolescentes peruanos 2009-2010.

\begin{tabular}{|c|c|c|c|c|c|c|c|c|c|c|c|c|}
\hline \multirow{3}{*}{$\begin{array}{c}\text { Estado } \\
\text { nutricional }\end{array}$} & \multicolumn{4}{|c|}{ Masculino } & \multicolumn{4}{|c|}{ Femenino } & \multicolumn{4}{|c|}{ Total } \\
\hline & \multicolumn{2}{|c|}{ MUST (NCHS) } & \multicolumn{2}{|c|}{ OMS } & \multicolumn{2}{|c|}{ MUST (NCHS) } & \multicolumn{2}{|c|}{ OMS } & \multicolumn{2}{|c|}{ MUST (NCHS) } & \multicolumn{2}{|c|}{ OMS } \\
\hline & $\%$ & IC95\% & $\%$ & IC95\% & $\%$ & IC95\% & $\%$ & IC95\% & $\%$ & IC95\% & $\%$ & IC95\% \\
\hline Déficit & 3,3 & $(2,9-3,7)$ & 0,3 & $(0,2-0,5)$ & 2 & $(1,7-2,3)$ & 0,2 & $(0,1-0,3)$ & 2,6 & $(2,4-2,9)$ & 0,2 & $(0,2-0,3)$ \\
\hline Peso bajo & 7 & $(6,5-7,6)$ & 1,2 & $(1,0-1,5)$ & 4,8 & $(4,3-5,3)$ & 1 & $(9,8-1,2)$ & 5,9 & $(5,6-6,3)$ & 1,1 & $(0,9-1,3)$ \\
\hline Normal & 78,5 & $(77,6-79,5)$ & 83,5 & $(82,7-84,4)$ & 79,5 & $(78,5-80,4)$ & 81,2 & $(81,7-82,6)$ & 79 & $(78,3-79,6)$ & 82,6 & $(82,0-83,2)$ \\
\hline Sobrepeso & 7,7 & $(7,1-8,3)$ & 10,9 & $(10,2-11,6)$ & 10,9 & $(10,2-11,6)$ & 14,1 & $(13,3-14,9)$ & 9,3 & $(8,8-9,8)$ & 12,5 & $(12,0-13,1)$ \\
\hline Obesidad & 3,5 & $(3,0-3,9)$ & 4 & $(3,5-4,4)$ & 2,2 & $(2,5-3,7)$ & 3,1 & $(2,7-3,4)$ & 3,2 & $(2,9-3,5)$ & 3,5 & $(3,2-3,8)$ \\
\hline
\end{tabular}

\section{RESULTADOS}

En la tabla 1 se puede observar el diagnóstico nutricional de acuerdo a dos referencias diferentes, la de Must $(\mathrm{NCSH})$ y la de la OMS. Esta última identifica mayor prevalencia de sobrepeso $(12,5 \%)$ y de obesidad $(3,5 \%)$ que la primera $(9,3$ y $3,2 \%$, respectivamente). En cuanto al déficit $(2,6 \%)$ y peso bajo $(5,9 \%)$ sucede todo lo contrario; es la de Must la que da las mayores prevalencias frente a la OMS, con 0,2 y $1,1 \%$, respectivamente.
El género femenino presentó mayor prevalencia de sobrepeso/obesidad $(13,1 \%)$ que el masculino (11,2\%). En lo que respecta al déficit/peso bajo, el género masculino fue el más afectado $(10,3 \%)$ (Must NCSH). En cuanto a la OMS, el sobrepeso/obesidad afectó más al género femenino $(17,2 \%)$ que al masculino (11,3\%). En cuanto al déficit/peso bajo, la prevalencia en el género masculino fue $1,5 \%$ y en el femenino $1,2 \%$.

La tabla 2 muestra el estado nutricional en relación a las variables es- tudiadas: área de residencia, nivel de pobreza, dominios geográficos y nivel de altitud. Los datos se presentan como prevalencias con su IC al 95\%.

El sobrepeso $12,2 \%$ (IC 11,5 a 12,9) y la obesidad 5\% (IC 4,6 a 5,6) fueron mayores en los adolescentes que vivían en áreas urbanas, mientras que el déficit $6,1 \%$ (IC 5,5 a 6,7 ) y el peso bajo 2,7\% (IC 2,4 a 3,1) predominó en las áreas rurales.

Los considerados no pobres presentaron las prevalencias más altas de

Tabla 2. Distribución de las características de los adolescentes según estado nutricional.

\begin{tabular}{|c|c|c|c|c|c|c|c|c|c|c|c|c|}
\hline Características & $\mathrm{N}^{0}$ & \multicolumn{2}{|c|}{ Déficit } & \multicolumn{2}{|c|}{ Peso bajo } & \multicolumn{2}{|c|}{ Normal } & \multicolumn{2}{|c|}{ Sobrepeso } & \multicolumn{2}{|c|}{ Obesidad } & $\mathrm{p}$ \\
\hline Área de residencia & & & & & & & & & & & & $<0,05$ \\
\hline Urbano & 7912 & 2,5 & $(2,2-2,9)$ & 5,8 & $(5,2-6,2)$ & 74,4 & $(73,5-75,4)$ & 12,2 & $(11,5-12,9)$ & 5,0 & $(4,6-5,6)$ & \\
\hline Rural & 6891 & 2,7 & $(2,4-3,1)$ & 6,1 & $(5,5-6,7)$ & 84,2 & $(83,4-85,1)$ & 5,9 & $(5,3-6,5)$ & 1,0 & $(0,8-1,3)$ & \\
\hline Nivel de pobreza & & & & & & & & & & & & $<0,05$ \\
\hline Pobreza extrema & 2366 & 3,5 & $(2,7-4,3)$ & 6,9 & $(5,9-8,0)$ & 84,1 & $(82,6-87,5)$ & 5,1 & $(4,2-6,0)$ & 0,4 & $(0,2-0,7)$ & \\
\hline Pobre no extremo & 4142 & 3,0 & $(2,5-3,5)$ & 6,8 & $(6,1-7,6)$ & 81,3 & $(80,1-82,5)$ & 6,8 & $(6,1-7,6)$ & 2,1 & $(1,6-2,5)$ & \\
\hline No pobre & 8295 & 2,2 & $(1,9-2,5)$ & 5,2 & $(4,7-5,7)$ & 76,4 & $(75,4-77,3)$ & 11,7 & $(11,1-12,4)$ & 4,5 & $(4,1-5,0)$ & \\
\hline Dominios & & & & & & & & & & & & $<0,05$ \\
\hline Costa norte & 1938 & 3,7 & $(2,8-4,5)$ & 6,7 & $(5,6-7,8)$ & 73,1 & $(71,1-75,0)$ & 11,9 & $(10,4-13,4)$ & 4,6 & $(3,7-5,5)$ & \\
\hline Costa centro & 880 & 1,7 & $(1,0-2,6)$ & 5,5 & $(4,1-7,0)$ & 71,3 & $(68,1-74,1)$ & 13,8 & $(11,6-16,0)$ & 7,8 & $(6,3-9,1)$ & \\
\hline Costa sur & 675 & 1,5 & $(0,7-2,5)$ & 4,3 & $(2,8-5,8)$ & 65,2 & $(61,8-69,2)$ & 17,9 & $(14,8-20,7)$ & 11,1 & $(8,6-13,5)$ & \\
\hline Sierra norte & 1212 & 2,5 & $(1,7-3,4)$ & 5,1 & $(4,0-6,4)$ & 84,5 & $(82,3-86,5)$ & 7,2 & $(5,8-8,8)$ & 0,7 & $(0,2-1,2)$ & \\
\hline Sierra centro & 3116 & 2,9 & $(2,3-3,5)$ & 7,0 & $(6,1-7,8)$ & 83,4 & $(82,0-84,7)$ & 5,7 & $(5,0-6,5)$ & 1,0 & $(0,7-1,4)$ & \\
\hline Sierra sur & 2145 & 2,9 & $(2,2-3,6)$ & 6,6 & $(5,7-7,7)$ & 81,3 & $(79,6-82,8)$ & 7,6 & $(6,5-8,8)$ & 1,6 & $(1,1-2,2)$ & \\
\hline Selva & 3608 & 2,3 & $(1,8-2,8)$ & 5,8 & $(5,1-6,6)$ & 82,0 & $(80,8-83,3)$ & 7,6 & $(6,7-8,5)$ & 2,3 & $(1,8-2,8)$ & \\
\hline Lima Metropolitana & 1229 & 2,4 & $(1,5-3,3)$ & 3,2 & $(2,3-4,1)$ & 71,8 & $(69,2-74,3)$ & 16,1 & $(14,0-18,2)$ & 6,5 & $(5,2-7,5)$ & \\
\hline Nivel de altitud (msnm) & & & & & & & & & & & & $<0,05$ \\
\hline 0 a 999 & 7672 & 2,6 & $(2,2-2,9)$ & 5,5 & $(5,0-6,0)$ & 75,7 & $(74,7-76,7)$ & 11,4 & $(10,7-12,1)$ & 4,8 & $(4,4-5,3)$ & \\
\hline 1000 a 2999 & 3344 & 2,1 & $(1,6-2,6)$ & 5,6 & $(4,8-6,4)$ & 82,1 & $(80,8-83,3)$ & 8,3 & $(7,4-9,2)$ & 2,0 & $(1,5-2,0)$ & \\
\hline 3000 a más & 3787 & 3,2 & $(2,7-3,9)$ & 7,1 & $(6,3-8,0)$ & 83,0 & $(81,7-84,2)$ & 5,8 & $(5,0-6,5)$ & 0,9 & $(0,6-1,2)$ & \\
\hline
\end{tabular}


Tabla 3. Análisis bivariado entre algunas características y estado nutricional.

\begin{tabular}{|c|c|c|c|c|}
\hline \multirow[t]{2}{*}{ Características } & \multicolumn{2}{|c|}{ Déficit/peso bajo } & \multicolumn{2}{|c|}{ Sobrepeso/obesidad } \\
\hline & $\mathrm{OR}$ & IC $95 \%$ & OR & IC $95 \%$ \\
\hline \multicolumn{5}{|l|}{ Género } \\
\hline Masculino & 1,57 & $(1,34-1,85)$ & 0,91 & $(0,79-1,05)$ \\
\hline Femenino & 1 & & 1 & \\
\hline \multicolumn{5}{|l|}{ Área de residencia } \\
\hline Urbano & 0,97 & $(0,83-1,14)$ & 3,1 & $(2,65-3,62)$ \\
\hline Rural & 1 & & 1 & \\
\hline \multicolumn{5}{|l|}{ Nivel de pobreza } \\
\hline Pobre extremo & 1,48 & $(1,14-1,93)$ & 0,29 & $(0,23-0,37)$ \\
\hline Pobre no extremo & 1,29 & $(1,08-1,56)$ & 0,58 & $(0,47-0,70)$ \\
\hline No pobre & 1 & & 1 & \\
\hline \multicolumn{5}{|l|}{ Dominios } \\
\hline Costa norte & 1,78 & $(1,24-2,56)$ & 0,73 & $(0,57-0,93)$ \\
\hline Costa centro & 1,46 & $(0,98-2,19)$ & 0,99 & $(0,76-1,28)$ \\
\hline Costa sur & 1,16 & $(0,71-1,89)$ & 1,44 & $(1,15-1,81)$ \\
\hline Sierra norte & 1,25 & $(0,85-1,83)$ & 0,3 & $(0,22-0,40)$ \\
\hline Sierra centro & 1,59 & $(1,16-2,18)$ & 0,27 & $(0,22-0,35)$ \\
\hline Sierra sur & 1,41 & $(0,99-1,98)$ & 0,4 & $(0,31-0,51)$ \\
\hline Selva & 1,3 & $(0,93-1,82)$ & 0,34 & $(0,27-0,42)$ \\
\hline Lima Metropolitana & 1 & & 1 & \\
\hline \multicolumn{5}{|l|}{ Nivel de altitud (msnm) } \\
\hline 0 a 999 & 0,83 & $(0,68-0,99)$ & 2,88 & $(2,35-3,53)$ \\
\hline 1000 a 2999 & 0,84 & $(0,68-1,04)$ & 1,45 & $(1,15-1,82)$ \\
\hline 3000 a más & 1 & & 1 & \\
\hline
\end{tabular}

sobrepeso $11,7 \%$ y obesidad $4,5 \%$ y los extremadamente pobres las mayores prevalencias de peso bajo $6,9 \%$ y de déficit 3,5\%.

En cuanto a los dominios, la obesidad se presentó más en la costa sur $11,1 \%$, costa centro 7,8\% y Lima Metropolitana $6,5 \%$. El sobrepeso en costa sur $17,9 \%$, Lima Metropolitana 16,1\% y costa centro $13,8 \%$. El peso bajo en sierra centro $7 \%$, costa norte $6,7 \%$ y sierra sur $6,6 \%$. El déficit, costa norte 3,7\%, sierra centro $2,9 \%$ y sierra sur $2,9 \%$.
Los que vivían por debajo de los 999 msnm tuvieron mayor prevalencia de sobrepeso $11,4 \%$ y de obesidad $4,8 \%$, mientras que quienes se encontraban por encima de los $3000 \mathrm{msnm}$, mostraban más peso bajo $7,1 \%$ y déficit 3,2\%.

En la tabla 3 se puede observar los OR y sus respectivos IC 95\%, de acuerdo a su estado nutricional, aquellos que se encontraban por debajo del 15p (déficit y peso bajo) y los que estaban por encima del 85p (sobrepeso y obesidad), en relación a los considerados normales (15 a 85p). Esto se ha contrastado con las variables de estudio: género, área de residencia, nivel de pobreza, dominios y nivel de altitud. Para ello se ha utilizado la regresión logística multivariada de muestras complejas. Para el primer grupo se encontró como factores de riesgo el ser género masculino, la pobreza extrema y no extrema y la costa norte y sierra centro. Como factores de protección, a los que vivían en ciudades por debajo de $1000 \mathrm{msnm}$ y entre 1000 y 2000 msnm. En cuanto al grupo de sobrepeso/obesidad, los factores de riesgo fueron que vivieran en áreas urbanas, en costa sur, en un nivel de altitud menor a los $1000 \mathrm{msnm}$ y entre 1000 y 2999 msnm; y como factores de protección, los pobres extremos, los pobres no extremos y todos los dominios, a excepción de la costa sur.

En la tabla 4 se puede observar el promedio y el DE de la talla observada, por edad y género lo mismo que su correspondiente número. El promedio

Tabla 4. Distribución de la talla según edad y sexo.

\begin{tabular}{|c|c|c|c|c|c|c|c|c|}
\hline \multirow{2}{*}{ Edad } & \multicolumn{4}{|c|}{ Masculino } & \multicolumn{4}{|c|}{ Femenino } \\
\hline & $\mathrm{N}^{0}$ & Talla obs. $(\mathrm{cm})$ & Talla ref. $(\mathrm{cm})$ & Diferencia & $\mathrm{N}^{0}$ & Talla obs. $(\mathrm{cm})$ & Talla ref. $(\mathrm{cm})$ & Diferencia \\
\hline 10 & 840 & $132,8 \pm 6,5$ & 139,9 & 7,1 & 803 & $133,4 \pm 7,9$ & 140,9 & 7,5 \\
\hline 11 & 754 & $137,7 \pm 7,5$ & 145,8 & 8,1 & 802 & $139,4 \pm 8,1$ & 147,6 & 8,2 \\
\hline 12 & 786 & $143,1 \pm 8,6$ & 152,5 & 9,4 & 806 & $145,1 \pm 7,7$ & 154,1 & 9,0 \\
\hline 13 & 846 & $149,2 \pm 9,5$ & 159,3 & 10,1 & 838 & $148,3 \pm 6,4$ & 158,8 & 10,5 \\
\hline 14 & 811 & $155,7 \pm 8,1$ & 165,7 & 10,0 & 841 & $150,5 \pm 5,7$ & 161,1 & 10,6 \\
\hline 15 & 882 & $159,9 \pm 7,2$ & 171,1 & 11,2 & 827 & $151,8 \pm 6,0$ & 162,1 & 10,3 \\
\hline 16 & 796 & $162,1 \pm 6,6$ & 174,9 & 12,8 & 748 & $152,6 \pm 5,8$ & 162,7 & 10,1 \\
\hline 17 & 693 & $163,7 \pm 6,4$ & 176,7 & 13,0 & 679 & $153,2 \pm 5,6$ & 163,3 & 10,1 \\
\hline
\end{tabular}

Talla obs. $=$ Talla observada. Talla ref. $=$ Talla referencial. 
Tabla 5. Análisis bivariado entre algunas características y el retardo de crecimiento.

\begin{tabular}{|c|c|c|c|c|c|}
\hline Características & $\mathrm{N}^{\circ}$ & Prevalencia & IC $95 \%$ & OR & IC $95 \%$ \\
\hline \multicolumn{6}{|l|}{ Género } \\
\hline Masculino & 6346 & 28,6 & $27,3-29,5$ & 1 & \\
\hline Femenino & 6346 & 28,5 & $27,5-29,7$ & 1,05 & $0,95-1,16$ \\
\hline \multicolumn{6}{|l|}{ Área de residencia } \\
\hline Urbano & 6628 & 17,9 & $1,7-18,8$ & 1 & \\
\hline Rural & 6124 & 39,9 & $38,7-41,2$ & 3,17 & $2,81-3,56$ \\
\hline \multicolumn{6}{|l|}{ Nivel de pobreza } \\
\hline Pobreza extrema & 2175 & 47,1 & $45,1-49,3$ & 4,08 & $3,48-4,78$ \\
\hline Pobre no extremo & 3682 & 34,5 & $32,9-36,1$ & 1,8 & $1,53-2,11$ \\
\hline No pobre & 6895 & 19,4 & $18,5-20,3$ & 1 & \\
\hline \multicolumn{6}{|l|}{ Dominios } \\
\hline Costa norte & 1615 & 22,7 & $20,6-24,8$ & 2,28 & $1,74-2,99$ \\
\hline Costa centro & 758 & 16,9 & $14,4-19,7$ & 1,54 & $1,12-2,11$ \\
\hline Costa sur & 552 & 10,3 & $7,8-12,9$ & 0,95 & $0,65-1,38$ \\
\hline Sierra norte & 1080 & 38,8 & $35,8-41,6$ & 4,59 & $3,51-5,99$ \\
\hline Sierra centro & 2729 & 38,4 & $36,6-40,1$ & 4,27 & $2,48-5,49$ \\
\hline Sierra sur & 1855 & 29,8 & $27,7-32,1$ & 2,67 & $2,09-3,43$ \\
\hline Selva & 3109 & 29,8 & $28,2-31,5$ & 3,06 & $2,43-3,84$ \\
\hline Lima Metropolitana & 1054 & 12,6 & $10,5-14,7$ & 1 & \\
\hline \multicolumn{6}{|l|}{ Nivel de altitud (msnm) } \\
\hline 0 a 999 & 6514 & 22,6 & $21,5-23,7$ & 1 & \\
\hline 1000 a 2999 & 2887 & 31,6 & $29,8-33,2$ & 1,19 & $1,96-2,60$ \\
\hline 3000 a más & 3351 & 37,2 & $35,5-38,8$ & 2,26 & $1,64-2,02$ \\
\hline
\end{tabular}

de la talla fue ligeramente mayor en el género femenino hasta los 12 años. A partir de esa edad predominaron los promedios en el género masculino. Las diferencias del promedio de las tallas alcanzadas, en el presente estudio, fueron menores cuando se las comparó con lo que muestra la tabla referencial $^{(12)}$. Estas diferencias no fueron muy diferentes, para ambos géneros, hasta los 14 años, empezando a tener mayor diferencia a partir de los 15 años en el género masculino.

La tabla 5 muestra la prevalencia de retardo de crecimiento y su OR con sus correspondientes IC al 95\%. La prevalencia nacional fue $28,5 \%$. Las prevalencias mayores se encontró en las áreas rurales $(39,9 \%)$, en los considerados pobres extremos $(47,1 \%)$ y los no extremos $(34,5 \%)$, en los dominios de sierra norte $(38,8 \%)$, sierra centro $(38,4 \%)$, sierra sur $(29,8 \%)$ y selva $(29,8 \%)$; y en los que viven de 1000 a 2999 (31,6\%) y por encima de $3000 \mathrm{msnm}$ (37,2\%). Los factores determinantes de riesgo fueron vivir en áreas rurales, la pobreza extrema y la no extrema, en los dominios costa norte, costa centro, sierra norte, sierra centro, sierra sur y selva, y en los niveles de altitud 1000 a 2999 y por encima de $3000 \mathrm{msnm}$.

\section{DISCUSIÓN}

Hasta el año 2000, aproximadamente, todos los estudios relacionados al estado nutricional de los niños y adolescentes eran analizados con la población de referencia del $\mathrm{NCSH}{ }^{(12)}$, y para el caso específico del adolescente, la publicada por Must y col en función a la misma referencia ${ }^{(10)}$. A partir del año 2000 irrumpen nuevas referencias, como la del Center of Disease Control (CDC) ${ }^{(14)}$, del International Task for Obesity (ITFO) ${ }^{(15)}$ y últimamente la que publica la OMS el año $2005^{(11)}$, teniendo todas ellas un reconocimiento internacional. Sin embargo, hay que reconocer que existen muchas más pero que son de uso local.

El problema que se suscita, teniendo varias poblaciones de referencia, es que, al tratar de comparar sus resultados, no se puede llegar a conclusiones válidas. Si bien la mayoría acepta que los criterios diagnósticos son los mismos (85 a 95 percentil sobrepeso y obesidad por encima del 95p), los valores correspondientes a estos percentiles no son iguales, en razón de la referencia, y esto trae aparejado diferentes prevalencias, hecho debidamente confirmado por una serie de estudios incluso en el Perú ${ }^{(16)}$. Lo mencionado se confirma con los resultados del presente estudio, donde con la referencia de la OMS la malnutrición por 'déficit' (déficit y peso bajo) fue $1,3 \%$ mientras que con la de Must $8,5 \%$, mientras que la malnutrición por 'exceso' (sobrepeso y obesidad) fueron 16 y $12,5 \%$, respectivamente.

El análisis del presente trabajo está hecho fundamentalmente con la referencia de Must y col, con la finalidad de tener una visión evaluativa del estado nutricional, en función del tiempo, dado que el principal punto de comparación se va a realizar con los resultados que se dieron en la Encuesta de Nutrición del Poblador Peruano (ENPPE 1975) ${ }^{(4)}$, donde se utilizó la misma referencia, lo que permite llegar a conclusiones válidas.

En ese sentido la ENPPE ${ }^{(4)}$ encontró $6,6 \%$ de sobrepeso y $1,3 \%$ de obesidad, mientras que el déficit alcanzaba a $5,1 \%$ y el peso bajo a $8,2 \%$, siendo el grupo femenino el más afectado. En el presente estudio, las prevalencias fueron 9,3 y $3,2 \%$ para el sobrepeso y la obesidad y 2,6 y $5,9 \%$ para el déficit y peso bajo, respectivamente. Por lo expuesto, se puede decir que el sobrepeso 
y la obesidad han incrementado y el déficit y peso bajo han disminuido. El año 75 predominaba el déficit y peso bajo $(13,3 \%)$ frente a su contraparte $(7,9 \%)$, mientras que en el presente estudio es al revés, el sobrepeso y la obesidad priman, $12,5 \%$ frente a $7,5 \%$. Las tendencias observadas en el presente trabajo que se dan en función del tiempo son consistentes con lo encontrado en el Brasil (17). Estos cambios responden a una serie de variaciones que se han suscitado en el campo económico, demográfico, epidemiológico, sociocultural, que se encuentran íntimamente relacionados entre sí y que de alguna manera son responsables de los estilos de vida que trae aparejado cambios en los patrones alimentarios y en la actividad física, proceso que se conoce como transición nutricional.

Esta tendencia que se ha observado en los adolescentes, también se ha observado en los niños preescolares ${ }^{(18)} \mathrm{y}$ en el grupo de 6 a 9 años ${ }^{(19)}$. En adolescentes argentinos, en el periodo 1995 a 2000 el sobrepeso ha pasado de 12,1 a $18,5 \%$ y la obesidad de 5,5 a $11,4 \%$, respectivamente ${ }^{(20)}$.

En un estudio en niños mexicanos de 10 a 13 años, utilizando la misma referencia encontraron un 2,6\% de déficit y $29,4 \%$ de sobrepeso (IMC > 85p) ${ }^{(21)}$. Una realidad diferente se da en Nigeria, donde mencionan $6,4 \%$ de déficit, $6,3 \%$ de sobrepeso y $1,8 \%$ de obesidad ${ }^{(2)}$. En el Ecuador hallan 13,7\% de sobrepeso y $7,5 \%$ de obesidad ${ }^{(22)}$.

En estudios en Argentina, utilizando la referencia del ITFO, el sobrepeso alcanzó $10,9 \%$ y la obesidad $2,2 \%{ }^{(23)}$ y $20,8 \%$ de sobrepeso y $5,4 \%$ de obesidad ${ }^{(24)}$. Con la misma referencia, la Encuesta Nacional de Salud y Nutrición en México señala que, en los varones, 21,2 y $10 \%$ presentan sobrepeso y obesidad, mientras que en las mujeres 23,3 y 9,2\%, respectivamente ${ }^{(25)}$. En África del Sur señalan la mayor presencia de sobrepeso y obesidad, siendo las más afectadas las mujeres (26).

En Colombia, con la referencia del CDC encontraron 2,5\% de delgadez,
11,3 de sobrepeso y $3,1 \%$ de obesidad (27). En la India, con la referencia de la OMS hallaron 9,8\% de sobrepeso y $4,8 \%$ de obesidad ${ }^{(28)}$, mientras que en Rusia el déficit y peso bajo fue 3,6\%, el sobrepeso $10,3 \%$ y obesidad $4,7 \%{ }^{(29)}$.

Por lo expuesto, se puede observar que, independientemente de la referencia utilizada, el problema de mayor magnitud que confronta el adolescente es el sobrepeso/obesidad. Esto implica que el riesgo de complicaciones es mayor, hecho ya documentado en lo que se refiere a la presencia del síndrome metabólico (SM) ${ }^{(30,31)}$, de resistencia a la insulina (RI) ${ }^{(31,32)}$ y dislipidemias ${ }^{(32)}$. Todas estas complicaciones comprometen muy seriamente su vida futura, dada su aparición a tan temprana edad.

El sobrepeso y la obesidad, en su conjunto, es más prevalente en el género femenino; estas diferencias podrían explicarse por cuanto las necesidades energéticas difieren entre varones y mujeres y también en relación a la tasa de crecimiento; por otro lado, los varones son más activos físicamente ${ }^{(33)}$. Otro condicionante tiene que ver con la imagen del cuerpo, en especial en las mujeres, que pueden conducir a comportamientos alimentarios que pueden resultar en ganancia de peso ${ }^{(34)}$.

Las diferencias en el IMC entre sexos puede ser el resultado del incremento de la masa grasa en las mujeres en contraste a los varones, quienes estabilizan su masa grasa y amplían su masa libre de grasa ${ }^{(35)}$. El hecho que los varones son más altos que las mujeres puede haber contribuido a que IMC sea más bajo, ya que la talla es el denominador. También disminuye la actividad física en las mujeres, lo que puede contribuir a un mayor depósito de grasa y aumento de peso ${ }^{(36)}$.

Las expresiones de malnutrición tienen entornos diferentes. En ese sentido, las variables asociadas al déficit/peso bajo y que de alguna manera fueron determinantes, son el género masculino, los considerados pobres extremos, los pobres no extremos, los que viven en costa norte y los de sierra centro. La única variable protectora fue el vivir por debajo de los $1000 \mathrm{msnm}$.

En cuanto al sobrepeso/obesidad, las variables que presentaron asociación y riesgo fueron el vivir en áreas urbanas, en la costa sur, por debajo de los 1000 msnm y los que estaban entre 1000 y 2999 msnm; mientras que las variables protectoras fueron pobreza extrema, pobreza no extrema, costa norte, sierra norte, sierra centro, sierra sur y selva.

De todas maneras, estos entornos no representan situaciones independientes, sino que de alguna manera pueden interrelacionarse, lo que explicaría la coexistencia de estos problemas en los mismos ámbitos geográficos ${ }^{(19)}$ o en la misma persona ${ }^{(37)}$.

Estudios anteriores mostraron que la costa sur es el que presenta la mayor prevalencia de obesidad ${ }^{(19)}$ y que departamentos como Moquegua y Tacna, que pertenecen a la costa sur, muestran lo mismo ${ }^{(5)}$.

El presentar los promedios de la talla alcanzada responde a tener un acercamiento a la realidad socioeconómica de un país, dado que la talla es considerada no solamente como un indicador biológico sino fundamentalmente como indicador resumen de las condiciones sociales en las que ha crecido un niño; es en definitiva un indicador global de calidad de vida. La talla final de un sujeto viene determinada en los genes y su adecuada expresión depende de factores ambientales.

Esto se ve reflejado de alguna manera en un estudio en Bangladesh, donde el promedio de talla en mujeres de 12 años de edad fue $137,1 \mathrm{~cm}$ y en varones de 16 años 154,2 cm. Si los comparamos con los nuestros (145,1 y $162,1 \mathrm{~cm}$, respectivamente), vemos cuán deprivados se encuentran en ese país ${ }^{(38)}$. Pero, si nos comparamos con países como EE UU ${ }^{(39)}$, la talla en mujeres de 12 años es $1,57 \mathrm{~m}$ y en varones de 16 años 1,76 $\mathrm{m}$; observamos las tremendas desigualdades que existen. En Corea, en un estudio nacional, de 10 a 19 años, el promedio de talla en el varón alcanzó $163,5 \mathrm{~cm}$ y en la mujer $156,8 \mathrm{~cm}^{(40)}$. 
Si nuestros datos los comparamos con lo informado por el Ministerio de Educación ${ }^{(5)}$, se observa que el promedio de la talla ha mejorado y esto es un reflejo de que las condiciones socioambientales van en esa misma dirección. De todas maneras esto hay que tomarlo con cierta cautela, por cuanto estos estudios han sido hechos en base a diseños metodológicos diferentes. Sin embargo, lo encontrado para los grupos preescolar ${ }^{(18)}$ y escolar ${ }^{(19)}$ nos alientan a pensar que los adolescentes también han mejorado.

La talla alcanzada a una determinada edad tiene que ver necesariamente con la prevalencia del retardo de crecimiento. Los países europeos que presentan mejores tallas que las nuestras hallan prevalencias que están por debajo del $4 \%{ }^{(41)}$. La tendencia que se observa en la mayoría de países es que estas prevalencias van disminuyendo ${ }^{(42)} \mathrm{y}$ por ende los promedios van mejorando. El porcentaje de disminución de la prevalencia está en razón de las mejoras sociales y económicas ${ }^{(43)}$.

En lo que se refiere al retardo de crecimiento, los determinantes de riesgo estuvieron relacionados a la ruralidad, la pobreza extrema y no extrema y la altitud 1000 a 2999 msnm y 3000 y más. En cuanto a los dominios, todos a excepción de la costa sur representaron factores de riesgo.

En conclusión, el sobrepeso y la obesidad fueron de mayor magnitud que el déficit y peso bajo, habiendo sufrido un incremento en función del tiempo, siendo los factores determinantes de riesgo el vivir en áreas urbanas, en la costa sur y por debajo de los 3000 msnm. Mientras que los factores de protección fueron estar en las categorías de pobreza extrema y no extrema y vivir en la costa norte, sierra norte, sierra centro, sierra sur y selva. La talla alcanzada dista de ser satisfactoria y refleja que aún mantenemos necesidades básicas insatisfechas. Las mayores prevalencias de retardo de crecimiento se dieron en los adolescentes pobres y en quienes vivían en la Sierra.

\section{REFERENCIAS BIBLIOGRÁFICAS}

1. World Health Organization/UNICEF. A picture of Health: a review of annotated bibliography of health of young people in developing countries. Geneva WHO 1995

2. Adesina A, Peterside O, Anochie I, Akani N Weight status of adolescents in secondary schools in Port Harcourt using body mass index (BMI). Italian J Ped. 2012;38:31-7. doi: 10.1186/18247288-38-31.

3. Instituto Nacional de Estadistica e Informática (INEI). Perú: Estimaciones y Proyecciones de Población 1950-2050

4. Pajuelo J. La obesidad en el Perú. Cuadernos de Nueva Perspectiva. Alimentación y Nutrición No. 1. Lima 1997.

5. Pajuelo J, Acevedo M. Situación nutricional de niños de 8 a 14 años en el Perú. Consensus. 2006;11(1):9-16.

6. Pajuelo J, Mosquera Z, Quiroz R, Santolalla M. El sobrepeso y la obesidad en adolescentes. Diagnostico. 2003;42(1):17-22.

7. Guo S, Chumlea W. Tracking of body mass index in children in relation to overweight in adulthood. Am J Clin Nutr. 1999;70:145S-8S.

8. Centro Nacional de Alimentación y Nutrición (CENAN-INS-MINSA). Instituto Nacional de Estadística e Informática (INEI). Informe del Estado Nutricional en el Perú. Componente Nutricional ENAHO-CENAN 2009-2010. Lima 2011.

9. Instituto Nacional de Salud (INS). Centro Naciona de Alimentación y Nutrición (CENAN). La medición de la talla y el peso. Guía para el personal de salud del primer nivel de atención. Lima INS 2004.

10. Must A, Dallal G, Dietz W. Reference data for obesity: 85th and 95th percentiles of body mass index (wt/ht2)-a correction. Am J Clin Nutr. 1991;54(5):773

11. De Onis M, Onyango A, Borghi E, Siyam A, Nishida C, Siekmann J. Development of a WHO growth reference for school-aged and adolescents. Bull World Health Org. 2007;85(9):660-7.

12. Organización Mundial de la Salud (OMS). Medición del cambio del estado nutricional. Ginebra 1983.

13. Instituto Nacional de Estadistica e Informática (INEI). Encuesta Nacional de Hogares sobre condiciones de vida y pobreza. Lima INEI 2012.

14. CDC growt charts. United States 2000. Disponible en: www.cdc.gov//growt.charts.

15. Cole T, Bellizi M, Flegal K, Dietz W. Establishing a standard definition for child overweight and obesity worldwide: international survey. BMJ. 2000;320:1240-3.

16. Pajuelo J, Medrano M. Utilización de diferentes poblaciones de referencia en el diagnóstico de los principales problemas nutricionales en niños y adolescentes. An Fac med. 2009;70(3):193-8.

17. Flores L, Gaya A, Petersen R, Gaya A. Trends of underweight, overweight, and obesity in Brazilian children and adolescents. J Pediatr Rio. 2013;89(5):456-61. doi: 10.1016/j. jped.2013.02.021.

18. Pajuelo J, Miranda M, Campos M, Sánchez J. Prevalencia de sobrepeso y obesidad en niños menores de cinco años en el Perú 2007-2010. Rev Peru Med Exp Salud Publica. 2011;28(2):222-7.

19. Pajuelo J, Sánchez Abanto J, Álvarez D, TarquiMamani C, Agüero R. Sobrepeso, obesidad y desnutrición crónica en niños de 6 a 9 años en Perú, 2009-2010. Rev Peru Med Exp Salud Publica. 2013;30(4):583-9.
20. Bejarano I, Dipierre J, Alfar E, Quispe Y, Cabrera G. Evolución de la prevalencia de sobrepeso y obesidad y desnutrición en escolares de San Salvador de Jujuy. Arch Argent Pediatr. 2005;103(2):101-9.

21. Ortiz L. Estado nutricional en adolescentes de una población suburbana de la ciudad de México. Rev Mex Ped. 2003;70(3):109-11.

22. Yepez R, Carrasco F, Baldeón M. Prevalencia de sobrepeso y obesidad en estudiantes adolescentes ecuatorianos del área urbana. Archivos Latinoamericanos de Nutrición. 2008;58(2):139-43.

23. Martinez C, Ibañez J, Paterno C, Semenza M, Itati M y col. Sobrepeso y obesidad en niños y adolescentes de la ciudad de Corrientes. Asociación con factores de riesgo cardiovascular. Medicina (Buenos Aires). 2001;61:308-14

24. Kovalskys I, Bay L, Rausch C, Berner E. Prevalencia de obesidad en una población de 10 a 19 años en la consulta pediátrica. Arch argent pediatr. 2003;101(6).

25. Shamah-Levy T, Villapando-Hernández S, Rivera S. Resultados de la ENSANUT 2006. Instituto Nacional de Salud Pública de México 2007.

26. Kimani-Murage E, Kanh K, Pettifor J, Tollman $\mathrm{S}$, Dunger D, et al. The prevalence of stunting, overweight and obesity, and metabolic disease risk in rural South African children. Public Health. 2010;10:158-70. doi: 10.1186/1471-2458-10-158.

27. Gamboa M, López B, Quintero C. Prevalencia de sobrepeso y obesidad en adolescentes del Municipio de Floridablanca, Colombia. Med UNAB. 2007;10(4):5-12.

28. Nawab T, Khan Z, Khan I, Ansari M. Influence of behavioral determinants on the prevalence of overweight and obesity among school going adolescents of Aligarh. Indian J Public Health. 2014;58(2):121-4. doi: 10.4103/0019557 X.132289.

29. Khasnutdinova S, Grjibovski A. Prevalence of stunting, underweight, overweight and obesity in adolescents in Velsk district, north-west Russia: A cross-sectional study using both international and Russian growth references. Public Health. 2010;124(7):392-7. doi: 10.1016/j. puhe.2010.03.017

30. Pajuelo J, Bernui I, Nolverto V, Peña A, Zevillanos L. Síndrome metabólico en adolescentes con sobrepeso y obesidad. An Fac med. 2007;67(2):143-9.

31. Pajuelo J, Bernui I, Rocca J, Torres L, Soto I. Marcadores bioquímicos de riesgo cardiovascular en una población adolescente femenina con sobrepeso y obesidad. An Fac med. 2009;70(1):7-10.

32. Pajuelo J, Arbañil H, Sánchez González J, Gamarra D, Torres L, Pando R, Aguero R. Riesgo cardiovascular en población infantil con sobrepeso y obesidad. An Fac med. 2013;74(3):181-6.

33. Kruger R, Kruger S, Macintyle U. The determinants of overweight and obesity among 10-to-15-yesrsold school children in the North West Province, South Africa. Public Health Nutr. 2006;9(3):351-8.

34. Neumark-Sztainer D, Paxton S, Hannan P, Haines J, Story M. Does body satisfaction matter?. Five years longitudinal associations between body satisfaction and health behaviors in adolescents females and males. J Adolesc Health. 2006;39(2):244-51.

35. Benefice E, Caius N, Gamier D. Cross-cultural comparison of growth, maturation and adiposity indices in two contrasting adolescent population in rural Senegal (West Africa) and Martinique (Caribbean). Public Health Nutr. 2003;7:479-85. 
36. Wenthe P, Janz K, Levy S. Gender similarities and differences in factors associated with adolescent moderate-vigorous physical activity. Pediatr Exerc Sci. 2009;21:291-304.

37. Pajuelo J, Villanueva ME, Chávez J. La desnutrición crónica, el sobrepeso y la obesidad en niños de áreas rurales del Perú. An Fac med. 2009;61(3):201-6.

38. Bosch A, Baqui A, van Ginneken J. Early-life determinants of stunted adolescent girls and boys in Matlab, Bangladesh. J Health Popul Nutr. 2008;26(2):189-99.

39. U.S. Department of Health and Human Services, Centers for Disease Control and Prevention National Center for Health Services. Anthropometric reference data for children and adults: United Sates, 2003-2006.

40. Sing YH. The correlation of blood pressure with height and weight in Korean adolescents aged 10-19 years; The Korean National Health and Nutrition Examination Surveys (2009-2011). Korean J Pediatr. 2014 Jan;57(1):35-40. doi: 10.3345/ kjp.2014.57.1.35.

41. Yngve A, De Bourdeaudhuij I, Wolf A, Grjbovski A, Brug J, Due P, Ehrenblad B, et al. Differences in prevalence of overweight and stunting in 11-years old across Europe: The Pro Children Study. Eur J Public Health. 2007;18(2):126-30.

42. Armstrong M, Lambert M, Lambert E. Secular trends in the prevalence of stunting, overweight and obesity among South African children (1994-2004). Eur J Clin Nutr. 2011;65:835-40. doi: 10.1038/ ejcn.2011.46.

Artículo recibido el 14 de octubre de 2014 y aceptado para publicación el 15 de noviembre de 2014.

Conflictos de interés:

Los autores niegan cualquier conflicto de interés.

\section{Correspondencia:}

Dr. Jaime Pajuelo

Correo electrónico: japara18@yahoo.com 